Maria Giulia Dondero*

\title{
Du texte à la pratique : Pour une sémiotique expérimentale
}

\author{
https://doi.org/10.1515/sem-2017-0081
}

Résumé: Notre travail vise à poursuivre les travaux récents de Jacques Fontanille qui envisage, à côté d'une sémiotique du texte, le développement d'une sémiotique de la pratique. Les travaux de Fontanille et notamment Pratiques sémiotiques (2008) développent des propositions qui s'étaient déjà faites jour dans l'article " Êtes-vous arpenteurs ou somnambules ? L'élaboration d'une typologie comportementale des voyageurs du métro » (1990) de Jean-Marie Floch. Nous allons dans un premier temps reparcourir le débat concernant la relation entre une épistémologie du texte et une épistémologie de la pratique, ainsi que la question de l'énonciation (énonciation énoncée, énonciation en acte, praxis énonciative). Dans un deuxième temps, nous proposerons le concept de textualisation comme lieu de médiation entre texte et pratique : les textualisations (photographies, vidéos, prises de notes) permettraient de contrôler le déploiement de la pratique qui est, quant à elle, éphémère et insaisissable. Si les textualisations sont produites in vivo, la notation par contre est une visualisation de la pratique qu'y diffère : elle fonctionne plutôt comme une reconstruction ex-post de la totalité de la pratique mettant en scène les évènements saillants ainsi que la grammaticalisation des gestes et des échanges. Plus généralement, l'objectif de ce texte est d'interroger les objets de la sémiotique : quels sont les objets qu'elle peut se donner, sans trahir un principe d'immanence qui a caractérisé la sémiotique textuelle et sans rester enfermée dans une condition qui la rend inapte à analyser les pratiques et à répondre aux questionnements de l'actualité sociale et de l'orientation de la recherche en sciences humaines?

Mots-clés: pratique, texte, textualisation, énonciation, notation

Abstract: Our essay aims at continuing the work of Jacques Fontanille, who developed a Semiotics of Practice next to a Semiotics of Text. This work, and especially Pratiques sémiotiques (2008), takes up a problem already formulated

Je remercie chaleureusement Anne Beyaert-Geslin pour sa précieuse relecture.

*Corresponding author: Maria Giulia Dondero, Langues et littératures françaises et romanes / Centre de Sémiotique et Rhétorique, Fonds National de la Recherche Scientifique, University of Liège, Liège, Belgium, E-mail: mariagiulia.dondero@ulg.ac.be 
by Jean-Marie Floch in the article "Êtes-vous arpenteurs ou somnambules? L'élaboration d'une typologie comportementale des voyageurs du métro" (1990). We will initially retrace the debate about the epistemological relationship between text and practice, outlining the forms of enunciative praxis. Secondly, we propose the concept of "textualization" as mediation between text and action: textualizations (photographs, videos, notes) organize and represent practices, which are in themselves ephemeral and elusive. Finally, we will consider the visualization that we call "notation": this analytical form of mediation between text and practice does not have the same status of in-vivo textualizations, but it works like an ex-post reconstruction which highlights the main gestures and exchanges while performing their grammaticalization. A more general aim of this essay is an inquiry into the legitimate objects of textual semiotics: What are the objects that it can analyze without betraying its principle of immanence, but also without being unable to analyze practices and respond to topical questions on social issues and on the research in the human sciences?

Keywords: practice, text, textualization, enunciation, notation

\section{Introduction}

Cet article vise à problématiser et à poursuivre les travaux récents de Jacques Fontanille qui envisage, à côté d'une sémiotique du texte, le développement d'une sémiotique de la pratique.

Les travaux déployés à ce sujet par Fontanille à partir de 2004 tout au long du séminaire intersémiotique de Paris IV-Sorbonne (« Pratiques sémiotiques », 20042006) et suivis de différentes publications dont l'ouvrage Pratiques sémiotiques (2008), développent des propositions qui s'étaient déjà faites jour dans le travail de Jean-Marie Floch, notamment dans l'article « Êtes-vous arpenteurs ou somnambules ? L'élaboration d'une typologie comportementale des voyageurs du métro », contenu dans l'ouvrage Sémiotique, marketing, communication (1990).

Floch a sans doute été le premier parmi les élèves d'Algirdas Julien Greimas à esquisser une analyse des pratiques. Si l'étude de Floch a permis de prendre en considération des grandeurs discursives autres que la textualité, en étudiant des parcours-types des utilisateurs du métro, elle n’a pourtant pas problématisé le statut des pratiques par rapport aux textes et n'a qu'en partie explicité les choix méthodologiques lors des observations de ces pratiques. En effet, Floch n'autorise ses lecteurs à garder le contrôle des différents types de comptes rendus des actions, en dérobant les instruments qui ont permis de parvenir à la typologie d'utilisateurs proposée - ce qui nous empêche de vérifier la « correspondance » entre les pratiques effectuées par les utilisateurs de métro et la typologie formulée. 
Autrement dit, ce que Floch ne nous a pas fourni, ce sont les textualisations des pratiques qui sont censées faire la «traduction » entre les pratiques en acte et une organisation des données pouvant être soumise à l'analyse sémiotique.

L'amorce de travail ethnographique fait par Floch nous a ouvert une voie bien avant que la discussion sur les pratiques devienne centrale, au moins dans certains lieux de la sémiotique européenne post-greimassienne, tels qu'en Italie et en France.

Parallèlement aux travaux de Jacques Fontanille en 2004, en effet, plusieurs chercheurs italiens et notamment Pierluigi Basso Fossali, ${ }^{1}$ ont courageusement lancé la discussion au sein des groupes de travail où l'on relevait le défi d'une sémiotique post-greimassienne capable de rendre compte des pratiques sociales : dans ce cadre, contrairement à ce qui était le cas chez Floch, le problème de la légitimité de ce niveau d'analyse par rapport au textualisme greimassien a d'emblée été posé.

Ce que nous nous proposons de faire dans le présent travail est de problématiser l'écart entre les propositions théoriques d'une sémiotique des pratiques, notamment dues à Fontanille (2008), et les outils d'analyse - encore à fabriquer. En effet, la réflexion théorique n'a pas été suivie par des analyses portant sur de "bons corpus » : les ouvertures théoriques sur la pratique ainsi que les propositions sur les différents niveaux d'immanence ont fini par s'écraser sur des corpus littéraires ou, tout simplement, sur des textes accomplis et/ou exemplaires. Nous allons en revanche nous concentrer sur les différentes formes de textualisation de l'action et revenir sur la question des relations entre texte et pratique et plus précisément sur la relation, jamais abordée jusqu'à présent, entre pratique et textualisation des pratiques.

La distinction entre texte et textualisation est capitale et nous la développerons au travers de la description proto-analytique d'un corpus constitué par des pratiques professionnelles en collaboration, et notamment par des pratiques collectives de conception architecturale - que nous avons explorées collectivement tout au long d'un programme de recherche mené à l'Université de Liège de 2011 à 2014. ${ }^{2}$

Nous allons ainsi revenir sur le débat actuel portant sur l'énonciation, qui accompagne un possible changement de paradigme allant d'une sémiotique du

1 Voir Basso-Fossali (2006). Les sceptiques envers une sémiotique des pratiques sont nombreux en Italie, et nous nous limiterons à citer l'ouvrage de Gianfranco Marrone (2010) qui défend la textualité comme unique grandeur possible de l'analyse sémiotique.

2 Ces observations de terrain ont été menées par les participants au programme de recherches ARC Common (Actions de Recherche Concertées COMMON - Collaboration Médiatisée Multimodale Naturelle [2011-2014]). Nous tenons à remercier Pierre Leclercq, coordinateur du projet A.R.C. Common ainsi que les autres membres du consortium pour la mise en commun du corpus. 
texte vers une sémiotique décrivant le sens en acte et les pratiques, tant dans leur singularité (scènes) que dans leur répétition/croisement/succession (stratégies).

Pour affronter cet objectif, les questions que nous nous posons sont multiples : est-il suffisant d'étudier les textualisations produites in vivo lors du déploiement des pratiques, c'est-à-dire la prise de notes, la photographie, l'enregistrement vidéo ou est-il plutôt nécessaire de constituer une notation de l'action qui reconstruise la totalité de la pratique ex post et qui mette en scène les évènements saillants ainsi que la grammaticalisation des gestes, des échanges, des interventions graphiques, etc. à travers un regard global et en surplomb ? Comment ne pas manquer, au travers de ces textualisations ou notations de pratiques, l'immédiateté de l'acte ? Comment ne pas passer à côté du sens de l'attente et de l'hésitation qui sont au cœur des échanges en cours, ainsi que de l'opacité du sens dans la complexité de son être en acte - un " être en acte » dont les bords et les cadrages se renouvellent constamment?

\subsection{Prémisses théoriques sur la relation texte-pratique}

Notre objectif est de nous interroger sur les objets légitimes de la sémiotique contemporaine : quels sont les objets qu'elle peut se donner sans trahir un principe d'immanence et sans rester enfermée dans un textualisme qui la rend inapte à analyser les changements sociétaux ainsi qu'une multitude d'orientations de la recherche en sciences humaines $?^{3}$ Le danger de la persévérance dans la voie du textualisme est l'homogénéisation méthodologique et épistémologique de toute expérience.

Pour limiter ces risques d'homogénéisation, Jacques Fontanille (2008) distingue six types d'expérience : figurativité pour les signes, cohérence et cohésion interprétatives pour les textes-énoncés, corporéité pour l'objet, pratique pour la scène pratique, conjoncture pour la stratégie et enfin éthos pour les formes de vie. Dans sa théorie, ces six types d'expérience sont associés à six instances formelles ou plans d'immanence, hiérarchisés selon un système d'interfaces de pertinence (Tableau 1).

À ceux qui lui objectent que le principe d'immanence est trahi dès que l'on sort de l'analyse de la textualité institutionnellement figée (un tableau, un roman, un film, etc.), Fontanille répond que le principe d'immanence est le fondement de tous les plans d'immanence et, à propos du plan de la scène pratique, il affirme :

3 Au sujet des enjeux sociétaux contemporains, voir Fontanille (2015). 
Tableau 1 : Fontanille (2008 : 34).

\begin{tabular}{|c|c|c|}
\hline Type d’Expérience & Instances Formelles & Interfaces \\
\hline Figurativité & $\begin{array}{c}\text { Signes } \\
\downarrow\end{array}$ & Formants récurrents \\
\hline $\begin{array}{l}\text { Cohérence et cohésion } \\
\text { interprétatives }\end{array}$ & Textes énoncés & $\begin{array}{l}\text { Isotopies figuratives de l'expression } \\
\text { Dispositif d'énonciation/inscription }\end{array}$ \\
\hline Corporéité & $\begin{array}{c}\text { Objets } \\
\downarrow\end{array}$ & $\begin{array}{l}\text { Support formel d'inscription } \\
\text { Morphologie praxique }\end{array}$ \\
\hline Pratique & $\begin{array}{l}\text { Scènes prédicative } \\
\downarrow\end{array}$ & $\begin{array}{l}\text { Scène prédicative } \\
\text { Processus d'accomodation }\end{array}$ \\
\hline Conjuncture & Stratégie & $\begin{array}{l}\text { Gestion stratégique des pratiques } \\
\text { Iconosation des comportments } \\
\text { stratégiques }\end{array}$ \\
\hline Ethos et comportement & Formes de vie & Styles stratégiques \\
\hline
\end{tabular}

Le principe d'immanence est indissociable [...] de l'hypothèse d'une activité de schématisation et demodélisation dynamique interne aux sémiotiques-objets et est l'aire d'activité immanente de cette schématisation qui doit nous indiquer pour chaque cas les limites du domaine de pertinence et non une décision a priori et tactique qui se focaliserait sur le seul texte. (Fontanille $2008: 14$ )

La théorisation de cette schématisation dynamique, interne aux différentes sémiotiques-objets, vise à justifier un type d'immanence qui ne s'appuie pas sur les bords et les limites de la textualité mais sur une forme d'iconisation interne à la pratique, qui l'organise : cette forme d'iconisation peut, d'une certaine manière, être décrite comme une sorte de " coagulation » des forces en jeu qui, comme le dirait Jean-François Bordron, " prennent ensemble » (voir Bordron 2011, 2014) et font émerger des formants d'organisation.

Selon Fontanille, chaque énonciation en acte développe elle-même une activité de schématisation, voire une méta-sémiotique interne qui est en construction lors de la pratique et de son observation. La question qui se pose est alors la suivante : cette activité de schématisation, comment doit-elle être abordée lors de l'analyse ? Faut-il que l'analyse s'accommode du modus operandi de la production de l'« objet » qu'on postule signifiant, c'est-à-dire de la pratique ? L'analyse, doit-elle imiter l'être en acte de l'action, partager la même dynamique de l'action en prenant en compte non seulement les actions effectuées mais aussi celles qui n'étaient que possibles en 
épousant une approche archéologique ? Doit-elle rendre compte du temps, du rythme et du tempo de la pratique ? Et comment ? Nous essayerons dans ce qui suit de voir comment les différentes textualisations produites lors de l'observation de terrain (prises de notes, photos, enregistrements vidéo) démultiplient et complexifient les perspectives sur la pratique en acte.

\subsection{Prémisses méthodologiques pour l'analyse des pratiques}

Si Fontanille a relancé le débat sur le problème de l'immanence et sur les fondements de la discipline, son ouvrage n'a pourtant pas orienté la sémiotique vers l'étude linguistico-ethnographique des pratiques. ${ }^{4}$ La linguistique des interactions, ainsi que les travaux de Lucy Suchman, ont inspiré en France la méthode analytique de Jacques Theureau, initiateur de l'approche « Le cours d'action $"{ }^{5}$ proche de la théorie sémiotique peircienne, et longuement discutée par Fontanille (2008). Tout en se référant aux analyses des pratiques de Theureau, Fontanille n'a pourtant pas posé la question du rôle d'intermédiaire que les textualisations et les notations des pratiques assument dans l'analyse des actions. Ce manque est compréhensible par le fait que, dans Pratiques sémiotiques, Fontanille analyse des textes littéraires qui sont des récits de pratiques et non pas des pratiques en elles-mêmes : le problème fondamental de la textualisation ne s'est donc pas posé car les pratiques en acte étudiées dans l'ouvrage sont en effet des récits, donc des textes accomplis. Fontanille a mis en place un modèle d'analyse de l'action en acte sur des actions qui avaient déjà été textualisées dans des textes littéraires ... Nous souhaitons par conséquent commencer notre exploration en distinguant les textes qui mettent en scène des pratiques (ce sur quoi se focalise l'attention de Fontanille) des textualisations des pratiques (ce sur quoi nous focaliserons la nôtre).

La démarche de Fontanille consiste à partir des textes littéraires accomplis et à les " remettre en acte ", à en extraire le développement et le déploiement : cette démarche ne lui permet pas de se poser le problème de la textualisation/ notation de l'acte. En revanche, lorsqu'on étudie une « vraie » pratique qui se déroule sous nos yeux à un moment donné au sein d'une culture donnée, il faut se poser la question des types d'observation et de textualisation ou notation pertinents, et choisir ainsi la saisie des formes émergentes des actions de la

\footnotetext{
4 Nous nous référons aux analyses de la linguistique appliquée et de l'ethnométhodologie. Voir à ce propos Goodwin (1994, 2000). Voir aussi Mondada (2005) et Hutchins (1995).

5 http://www.coursdaction.fr/accueil.htm
} 
pratique, voire les grammaticalisations locales des composants de la pratique dans le temps (unités, règles d'agencement, répétitions, etc.).

Les textualisations des pratiques posent en fait le problème de ce qui est nécessaire d'observer et/ou de sélectionner de la pratique pendant qu'elle est en acte. Cette sélection s'opère aussi grâce aux propriétés technologiques des différents média participant à la saisie de l'émergence des formes des pratiques. Un de problèmes à affronter à ce propos est d'ailleurs celui de la commensurabilité entre les saillances et les discontinuités repérées par les différents médias utilisés dans la production des textualisations (photos, vidéos, notes). Ces textualisations pourraient d'ailleurs être conçues comme des protoanalyses : l'analyse finale devrait en fait également reparcourir le geste médiatique qui a sélectionné une certaine saisie de la pratique, pour en caractériser la spécificité, les contraintes technologiques ainsi que les contraintes liées au point de vue.

Outre la différence entre les textes qui mettent en scène des pratiques - et qui peuvent être éventuellement utiles pour étudier une idéologie de l'action et les textualisations de pratiques, il faut pouvoir distinguer également entre textualisation de la pratique et notation de la pratique. Les textualisations telles que l'enregistrement vidéo, la photographie, la prise de notes ne peuvent pas être définies comme des notations car elles demeurent trop " fidèles » à la pratique elle-même et à sa densité phénoménologique. Ce faisant, elles ne peuvent pas répondre aux critères de la notation qui vise la visualisation " d'en haut » de la totalité des actions, et qui permet de cartographier l'émergence de gestes, leurs durées, leurs croisements, leurs successions à partir d'un regard globalisant. La notation, en effet, ne se présente pas à l'instar d'un témoignage de la pratique mais bien au contraire comme une reconstruction de la pratique qui repère des homogénéités et des hétérogénéités parmi les configurations des gestes, les attaques, les moments de pause, le tempo de la durée, le type de participation corporelle des acteurs, etc. L'objectif en est l'identification de l'émergence de modules d'actions qui sont plus ou moins répétés, plus ou moins en contraste les uns avec les autres, plus ou moins fixes ou déformables et qui peuvent rendre visuellement évident un micro-alphabet des structures de l'action, ou au moins un ensemble de patterns. Pour ce faire, nous aborderons l'observation des pratiques de conception architecturale collaboratives afin d'explorer les différents degrés de densité phénoménologique témoignés par les textualisations des actions.

Avant d'aborder le corpus choisi, nous souhaitons rappeler les derniers avatars de la théorie de l'énonciation qui, tant dans sa déclinaison textualiste que dans ses déclinaisons plus phénoménologiques ou liées à la description des cultures, nous apparaît toujours au fondement de notre méthodologie 
disciplinaire. La stratégie méthodologique de la sémiotique textualiste pourra ainsi être mise en question car la perspective des textualisations de la pratique nous oblige à distinguer entre un parcours énonciatif de production d'un texte (qui relève d'une énonciation en acte), les simulacres de l'énonciation contenus dans le texte produit (qui relèvent d'une énonciation énoncée) et les pratiques de valorisation de ces textes au sein d'une culture donnée (domaine de la praxis énonciative).

\section{La théorie de l'énonciation dans le débat contemporain}

Ces quinze dernières années, les recherches sémiotiques ont en effet théorisé trois sortes de niveaux énonciatifs selon les degrés de stabilisation et de clôture de l'objet d'analyse, à savoir : l'énonciation énoncée (les simulacres de la subjectivité en discours), la praxis énonciative (les transformations/renouvellements internes à une culture et entre différentes cultures), l'énonciation en acte (un ensemble d'actions à saisir dans leur déploiement) ${ }^{6}$

Pour résumer brièvement le débat, surtout franco-italien, nous nous appuierons surtout sur les propositions de Claudio Paolucci exposées dans son ouvrage paru en 2010 qui a pour titre Strutturalismo e interpretazione (2010b) ainsi que sur les propositions faites par Fontanille (2014) en concomitance avec les réflexions de Bruno Latour (2012) formulées dans son ouvrage, Enquête sur les modes d'existence.

Si la théorie de l'énonciation peut être définie comme la théorie de la distribution de la subjectivité à l'intérieur du discours, dans son ouvrage, Paolucci prend position par rapport à cette tradition en rappelant que la subjectivité théorisée par Greimas et Courtés dans leur dictionnaire (1979), tout en se déclarant "énoncée ", voire concernant des simulacres textuels, garde, à la suite de Benveniste, le je-ici-maintenant comme source et repère de tout discours. Cela reviendrait à dire que les localisations déictiques maintiennent en vie un modèle d'analyse extralinguistique : celui de la situation de communication. De ceci découle, selon Paolucci, que la théorie sémiotique de tradition benvenistienne aurait péché en hiérarchisant le « je-tu » par rapport au « il » de la troisième personne. Cette "supériorité » du je-ici-maintenant, entendu comme source de toute prédication, sur la troisième personne, comprise comme résultat secondaire d'une relation je-tu in presentia, avait déjà été dénoncée par Giovanni Manetti (1998) comme une « brèche dans le structuralisme ». Autrement dit : le

6 Voir à ce propos Fontanille (2003). 
je-ici-maintenant, peut-il être considéré comme une survivance de transcendantalisme dans la théorie greimassienne ? Et encore : la théorie de l'énonciation, en tant que brèche dans le structuralisme, peut-t-elle bousculer l'immanence hjelmslevienne liée à la logique du texte ? L'immanence textuelle peut-elle encore être valorisée comme centrale si l'on situe la situation de communication comme source de la théorie greimassienne de l'énonciation - ce qui est pointé du doigt par Paolucci ?

La tradition sémiotique des déictiques et des simulacres, on le sait, se distingue du modèle de l'énonciation impersonnelle proposée par Christian Metz (1991), centré sur une subjectivité diffuse et non déictique, ainsi que de la sémiotique interprétative, peircienne, développée en Italie par Umberto Eco (1975, 2013 [1984]), Patrizia Violi (2006), et Claudio Paolucci (2010a, 2010b).

Il faut tout d'abord préciser que Metz, à la différence de l'école peircienne italienne, maintient une position textualiste (et spécifique du texte filmique). Quant à elle, la sémiotique interprétative italienne ne réduit pas l'énonciation impersonnelle à une propriété de l'énoncé mais utilise, sous l'élan de Paolucci, la notion d'énonciation impersonnelle pour concevoir la relation entre les différents plans d'immanence présents dans le fonctionnement de l'encyclopédiel'encyclopédie étant d'ailleurs un modèle de fonctionnement du sens disposé en rhizome où toute action régissant l'actualisation des réseaux de sens prend appui sur un événement énonciatif conçu comme impersonnel.

L'énonciation impersonnelle deviendrait ainsi le dispositif régulateur de la relation entre pratiques de donation et de génération du sens à l'intérieur d'une culture (Paolucci 2010b). Plus précisément, l'énonciation serait entendue en tant que dispositif de contrôle, d'orientation et de gestion des activités d'assimilation/ refus/transformation du neuf et de l'étranger dans les pratiques sociales quotidiennes - sur le modèle de la praxis énonciative qui, chez Fontanille (2003) et Fontanille et Zilberberg (1998), est déterminée par les modes d'existence : virtualisalisation, actualisation, réalisation, potentialisation.

\subsection{Enonciation impersonnelle et praxis énonciative}

Sur la question de l'énonciation impersonnelle, Paolucci (2010a) revient sur la logique des relatifs de Peirce, et affirme que l'événement est premier et les subjectivités qui éventuellement l'incarneraient sont secondes et occupent les positions actantielles ouvertes par l'événement. Paolucci appelle cette inversion de perspective " une tension vers la périphérie de l'encyclopédie » : on n'organiserait plus le langage en fonction des centres déictiques d'énonciation (je-icimaintenant). L'attention se déplacerait ainsi du sujet comme centre de la schizie 
créatrice (chez Greimas) aux positions variables des instances de la subjectivité dispersées dans l'encyclopédie et régies par les événements (le sujet devient ainsi chez Paolucci un " occupant sans position fixe »).

Paolucci (2010a) utilise non seulement la théorie de Metz pour concevoir une sémiotique des pratiques, mais également la théorie de l'acteur-réseau de Latour et affirme que les " effets de sujet " circulent comme des virtualités actantielles (humaines et non-humaines) organisant le sens à partir d'un mouvement premier identifié comme un évènement impersonnel : l'action.

Dans la théorie de la praxis énonciative chez Fontanille également, le point de vue est impersonnel (il s'agit de stocks de contenus qui se réalisent, actualisent, virtualisent et potentialisent réciproquement). La praxis énonciative se débraie en ce que Paolucci appelle un évènement, qui distribuera ensuite les positions actantielles pertinentes à l'étude de la signification. Ce changement de perspective implique que la source du débrayage n'est plus un sujet énonciateur mais l'événement en soi. L'événement, entendu comme une troisième personne impersonnelle, traverse les différents types de textes et d'institutions : la proposition de Paolucci concerne en effet une distribution de positions actantielles à partir des parcours/ événements au sein du rhizome encyclopédique.

Dans la théorie de Fontanille, non seulement au niveau général de la praxis énonciative, qui reformule la relation saussurienne langue-parole en une relation entre formes et opérations, mais aussi aux niveaux des pratiques et des formes de vie, les énonciations sont enchaînées et superposées les unes sur les autres et la singularité de tout acte énonciatif devient pertinente seulement dans un second temps.

\subsection{Enonciation impersonnelle et "sens en acte »}

Précisons cette notion d'événement impersonnel en revenant sur l'un des travaux récents de Fontanille (2014) ayant pour titre " L'énonciation pratique : exploration, schématisation et transposition » (2014), qui me semble ajouter un point de vue important à la théorie de Latour (2012) et à la sémiotique postpeircienne italienne.

Fontanille affirme que ses propositions sur l'étude des pratiques sont en partie inspirées des chaînes caractérisant chaque mode d'existence selon Latour (2012), et notamment de l'origine des chaînes, voire de l'énonciation/autoénonciation des modes d'existence. En épousant le même défi que Latour, à savoir la non-distinction préalable entre sujet et objet, Fontanille revient sur l'affirmation de Latour " qu'il y a "instauration” des modes d'existence, par 
intensification de leur présence, jusqu'à leur manifestation observable » (Fontanille 2014 : 3, nous soulignons).

Fontanille vise à caractériser cette intensification de la présence et à comprendre la manière dont la signification de la pratique émane d'elle-même à partir des formes d'agencement des cours d'action :

Les pratiques sont des cours d'action ouverts aux deux bouts de la chaîne, dont la classe thématique est identifiable à une détermination extérieure, mais dont l'« objet » (et par conséquent le « sujet » qui le vise) reste indéterminé et labile tout au long du cours d'action, tout au moins aussi longtemps que la signification de ce dernier n'est pas définitivement fixée, de l'intérieur de la pratique même, par les acteurs eux-mêmes. C'est pourquoi la signification des pratiques ne peut être élaborée à distance, parce que le point de vue distant et débrayé ne saisit rien qui relève en propre du "sens pratique ». (Fontanille 2014 : 2, nous soulignons)

Fontanille conçoit par conséquent une analyse en immersion (embrayée), où le caractère subjectal ne pourra apparaître qu'a posteriori : si tout était fixé dès le début (un sujet, un objet) alors on serait encore pris dans une perspective narrative et greimassienne. Mais comment expliquer cette intensification de présence qui nous amène d'un mouvement général de durée et de persistance de l'existence, vers la signification?

La signification d'une pratique émerge de l'intérieur de la pratique ellemême, par un parcours d'instauration, l'instauration étant la forme primaire de l'énonciation : « la signification émerge de la dynamique d'un cours d'action, et de l'activité d'une instance qui est elle-même en cours d'instauration » (Fontanille 2014 : 4). De ce mouvement d'instauration découle la recherche d'actants auxquels on pourrait imputer le franchissement des obstacles et la continuation du cours d'action. Mais au moment même de l'instauration, l'instance d'imputation reste diffuse, plurielle, et confond par définition, notamment, les acteurs eux-mêmes et l'analyste. Cette instauration, dit Fontanille, est bien une énonciation impersonnelle - sans objet ni sujet -, diffuse - sans identités posées a priori -, et en mouvement.

Pour expliquer l'émergence de formes et de positions actancielles à partir de cette énonciation impersonnelle, Fontanille revient à la praxis énonciative, elle aussi impersonnelle, qui agit par des opérations de tri hiérarchisant plusieurs potentiels de manifestation, plusieurs formes qui sont censées s'actualiser en en virtualisant d'autres. Mais ce principe d'instauration ne se suffit pas à lui-même et Fontanille propose de se servir de la notion d'" autoaffection » : l'expérience d'exister est un affect élémentaire, celui que l'existence se donne à elle-même. L'expérience, en somme, serait l'expérience de l'immersion dans l'existence. 
Fontanille articule cette expérience en deux moments : la réflexion et l'exploration. Dans la réflexion, on aperçoit deux pôles : le premier est affectant, le second est affecté, ce qui permet de concevoir un premier stade de la stabilisation de formes, voire des procédures d'iconisation assurant la reconnaissance des phases et des moments du cours pratique : "La réflexion forme et déforme des schémas et des modèles, et l'on peut considérer à cet égard que l'énonciation comporte une dimension de modélisation interne » de l'action (2014: 10).

Ce premier moment de la prise de forme de l'expérience est expliqué par Fontanille à travers le concept d'« épilinguistique » hérité d'Antoine Culioli qui permet d'installer dans l'énonciation une instance réflexive, subconsciente et diffuse, et qui néanmoins explore et régule les potentiels de signification des expressions en cours de formation.

La seconde articulation, l'exploration, concerne le déploiement de la réflexivité et s'étend de l'activité épilinguistique, interne à l'usage lui-même, à celle, finale, métalinguistique, propre à l'analyste qui explore la pratique de l'extérieur. Dans la phase de l'exploration, l'écart entre les rôles affectant et affecté augmente, les rôles actanciels sont distribués dans des acteurs distincts. L'exploration permet ainsi de dépasser les limites d'une instauration impersonnelle du sens et convoque à la fin du parcours allant de l'expérience pratique à l'analyse de la pratique les observateurs des pratiques qui sont censés développer l'activité épisémiotique des acteurs eux-mêmes. Fontanille précise à ce propos qu'il ne faudra pas ici supposer un niveau de pertinence différent du précédent et une instance d'observation débrayée, mais une complexification de la scène pratique elle-même, ainsi qu'un enrichissement systématique de ses procédures d'exploration et de sa dimension épisémiotique.

L'analyse des pratiques en immersion ferait donc partie de leur énonciationinstauration. Elle ne saurait se positionner en surplomb et dans une position méta-sémiotique préalable et $a$ priori sans rater ce qu'il y a de pratique dans le sens pratique. La position surplombante et méta-sémiotique sera accessible comme résultat final de l'analyse.

Il me semble que les approches de Paolucci (2010a et 2010b) et de Fontanille (2014) suivent le même schéma : on part d'un événement, voire d'un mouvement diffus, multiple, ${ }^{7}$ et par intensification de la présence et stabilisation des formes, on arrive à ce que Paolucci appelle "distribution des positions actancielles". Chez Paolucci, le fond de la pratique en acte est identifiable au travers des

7 Il s'agit de l'indicialité en tant que questionnement et virtualité chez Bordron (2011 et 2014), et de la « force qui s'exerce » chez Latour (2012). 
parcours exploratoires au sein de l'encyclopédie ; chez Fontanille, en revanche, il s'agit d'une praxis énonciative régie par les modes d'existence. Dans les deux cas, la trajectoire est orientée de l'événement impersonnel à la distinction entre subjectal et objectal.

\section{Le Studio Digital Collaboratif}

Venons-en au corpus : les pratiques de travail collectif, et plus précisément, la conception architecturale collaborative en présence et à distance.

Le groupe de recherche interfacultaire A.R.C. Common avec lequel nous avons travaillé à l'université de Liège de 2011 à 2014, constitué par des ingénieurs, des architectes, des psychologues du travail et des sémioticiens, a produit et ensuite soumis à l'analyse un studio digital collaboratif ${ }^{8}$ permettant aux architectes travaillant dans des villes lointaines de tracer ensemble, en temps réel, un même dessin en utilisant une tablette digitale partagée par les acteurs en jeu.

Le Studio Digital collaboratif (désormais : SDC) est un dispositif matériel qui associe un module externe de visio-conférence s'appuyant sur Skype et un bureau virtuel récréant les conditions de la communication en coprésence. Chaque trait tracé par un architecte sur la table virtuelle est immédiatement visualisé sur la table située dans le siège à distance, ce qui permet à l'autre architecte d'interagir immédiatement sur le trait proposé, de le supprimer, de le modifier, etc. Tout geste graphique sur la table est enregistré par la machine elle-même.

Dans notre démarche méthodologique, nous n'étudierons pas la manière dont ce trait graphique est manipulé ; en revanche nous enquêterons directement la pratique collective collaborative globale, qui se constitue en tant qu'événement impersonnel au sein d'une série d'habitudes et de procédures de travail (praxis énonciative).

Dans le cadre de cette pratique collaborative, nous pouvons repérer deux types de textualisation : les textualisations en acte tout au long de la pratique (et plus particulièrement la production des dessins par les architectes) ainsi que les textualisations produites par l'observateur/analyste à partir de la pratique de production des dessins des architectes, à savoir les photos, la vidéo, les notes. Ce deuxième type de textualisations fonctionne comme une médiation entre les

8 http://www.ulg.ac.be/cms/c_147500/fr/lucid-presente-le-studio-digital-collaboratif. Voir Elsen et Leclercq (2008) et Rajeb et Leclercq (2013). 
pratiques de dessin des architectes et les productions de l'observateur/analyste (photos, vidéos, notes).

Autrement dit, notre approche méthodologique propose en premier lieu l'étude des coagulations d'actions en des textes visuels, produits à l'intérieur d'une pratique professionnelle (les dessins, les esquisses, etc.) qui se stabilisent de manière plus ou moins rapide sur le support du SDC. Ces textualisations produites au sein de la pratique font l'objet, dans un deuxième temps, de textualisations verbales, photographiques et audiovisuelles de niveau supérieur (produites à partir de la pratique) qui les mettent en perspective : elles ajoutent la perspective de la prise de vue de l'observateur à la perspective de la production des dessins par les architectes.

Pour les observateurs/analystes situés au cœur du déroulement de la pratique, il s'agit de démêler et de débrouiller les différents types de «coagulation » du sens qui produisent les textualisations que nous appelons de niveau $n-1$ (les dessins et les annotations des architectes). Il faudra les étudier à travers les textualisations de la pratique (notes, photos, enregistrements vidéo) que nous appelons de niveau $n+1$, la pratique en acte étant le niveau de départ $n$.

La pratique en elle-même est un événement fuyant et non-répétable : elle possède un statut autonome par rapport aux textualisations qu'elle produit (n-1) et qui sont produites à partir d'elle $(n+1)$ : elle est une dynamique d'organisation du sens ouverte, que les différents niveaux de textualisation permettent partiellement de canaliser.

Prenons le cas d'un enregistrement vidéo d'une réunion de conception collaborative à distance (Figure 1).

Les deux cadrages en haut de la composition représentent les deux architectes occupés à la conception collaborative à distance. Ces deux images sont issues de la caméra installée sur l'ordinateur. Les deux images positionnées en bas représentent, à travers une perspective d'en haut, la table digitale qui est utilisée par les deux architectes et qui est manipulable en temps réel.

Les textualisations internes à la pratique $(n-1)$ sont identifiables au travers des dessins et des annotations que le chef d'atelier et les collaborateurs produisent plus ou moins collectivement (images d'en bas). Ces dessins sont textualisés à leur tour par les observateurs de la pratique à travers la photo, la prise de notes et ici, notamment, par la vidéo $(n+1)$. Ces textualisations $n+1$, que l'observateur produit et qui constituent des intermédiaires entre la pratique en acte et l'analyse future, sont des prothèses de la mémoire de l'observateur, qui visualisent et organisent de manière différente la " même » pratique et qui, plutôt que de la stabiliser et de la réifier comme définitive, la démultiplient à travers des perspectives médiatiques diverses. 


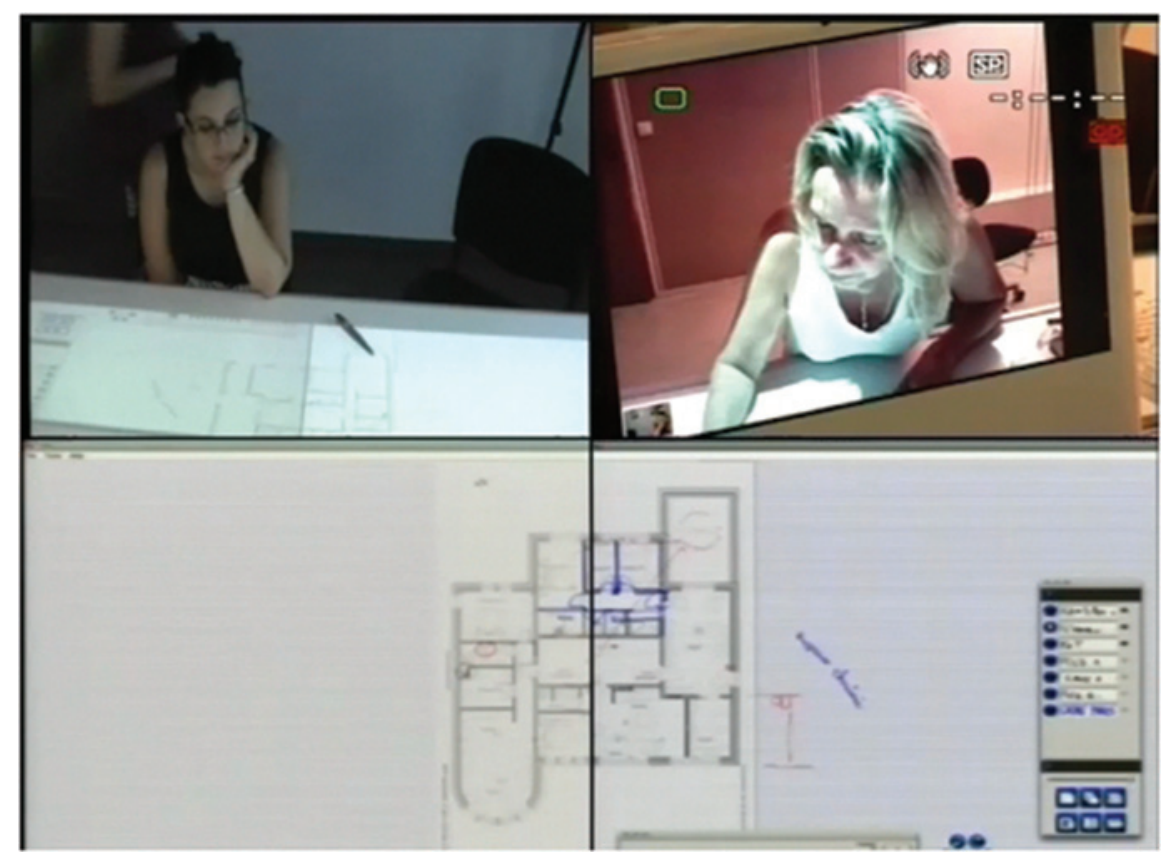

Figure 1 : Collation en un seul écran de la vision du bureau virtuel et de la vision des architectes au travail sur ce même bureau lors d'une réunion à distance (via Skype).

Si les dessins et annotations, produits par les architectes, sont les textualisations-cible de la pratique de conception ( $n-1)$, les textualisations vidéo, les photos et les notes, produites par l'observateur, sont en revanche les textualisationscible de la pratique analytique $(n+1)$.

Comment peut-on décrire la densité syntaxique de ces dessins $(n-1)$ par rapport à l'acte même qui les trace ? S'agit-il de la même densité ? Non, justement : les dessins sont moins denses que les gestes car ils rencontrent des critères d'inscription et de disposition sur le support qui les accueille. Les dessins « soustraient » de la densité phénoménologique aux actes de traçage, ces derniers étant censés canaliser les intentions des gestes, les soumettre aux règles et aux contraintes du plan d'expression et plus précisément du support formel (Fontanille 2005) ainsi qu'aux contraintes de la collaboration avec le collègue distant (correction/ajustement/suppression).

Les textualisations $n-1$, contrairement aux textes, sont encore manipulables et modifiables pendant la pratique en cours (et les textualisations $n+1$, d'ailleurs, les représentent in progress). En revanche, elles possèdent déjà un support qui les rendra suffisamment «stables » pour pouvoir être extraites, en tant que 
futurs textes, de la pratique de production, puis transportées vers d'autres pratiques (révision des collègues, etc.). Ceci dit entre parenthèses, nous considérons les conversations entre les architectes comme des textualisations de type différent des dessins : elles s'inscrivent dans l'espace acoustique de la pratique mais elles ne sont pas ancrées dans un support qui dure : leur caractère éphémère ne les rend pas aptes à être extraites de la pratique-source et transportées dans d'autres pratiques.

\section{Observations, textualisations, et notation}

Nous avons examiné le statut des textualisations $n-1$, à savoir les textualisations produites par la pratique de conception architecturale (les dessins et les annotations) ; venons-en aux textualisations qui sont en revanche produites par l'observation $(n+1)$.

Sur quelles textualisations pouvons-nous nous baser pour analyser une pratique de textualisation qui est à son tour l'objet de textualisation ? Comment mettre en rapport les rythmes de textualisations internes à la pratique (production de dessins) et les rythmes de textualisation de la pratique ? Autrement dit : comment mettre en relation les modalités de production des textualisations $n-1$ (gestes du traçage et production de dessins) avec les média qui les textualisent $n+1$ (notes, photos et vidéo)?

Nos observations de terrain ont précisément produit trois types de textualisation de la pratique de conception architecturale en présence ${ }^{9}$ :

1. Les notes, que nous définissons comme un type de textualisation caractérisé par la ponctuation et la linéarité : ponctuation au sens où les notes segmentent la pratique en en valorisant des moments-clés ou bien des tendances ; linéarité car le support de l'écriture et les règles qui les soutiennent en assurent une lecture linéaire de gauche à droite.

2. La photographie peut, surtout dans le cas de la collaboration en coprésence, rendre compte de la séance avec une fidélité majeure par rapport à la prise de notes mais elle peut se révéler, dans sa fonction proto-analytique, moins manipulable et moins flexible que l'écriture. Comme les notes, la photo peut arrêter des moments uniques de la pratique (Figure 2) ou bien les résumer dans une vision plus globale, surtout lorsqu'une action s'arrête sur un problème ou une hésitation (Figure 3).

9 Voir aussi Angenot et al. (2013). 


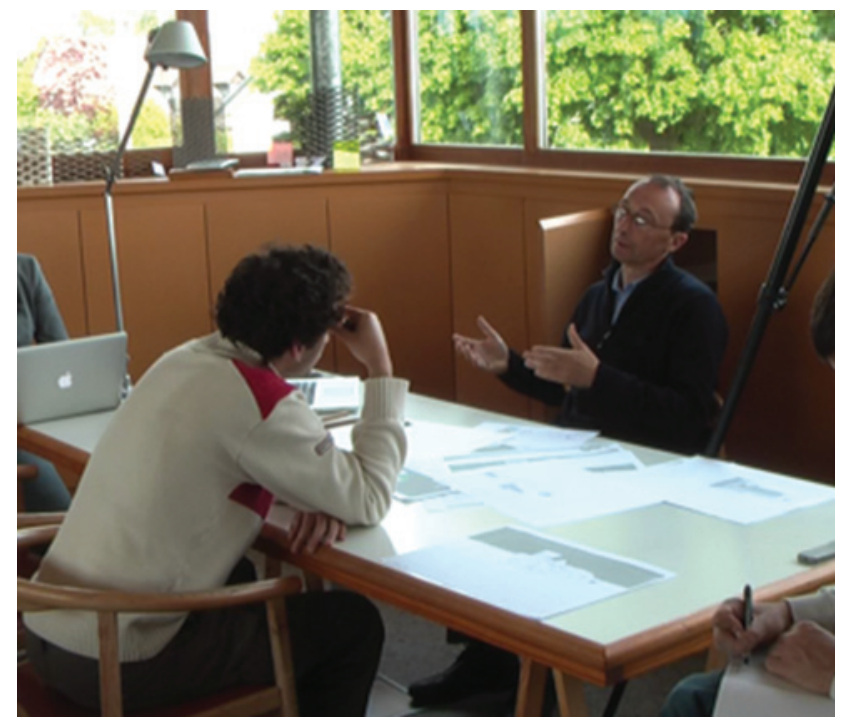

Figure 2 : Photo d'un moment saillant dans la discussion lors d'une réunion en coprésence dans un atelier d'architecture en Belgique.

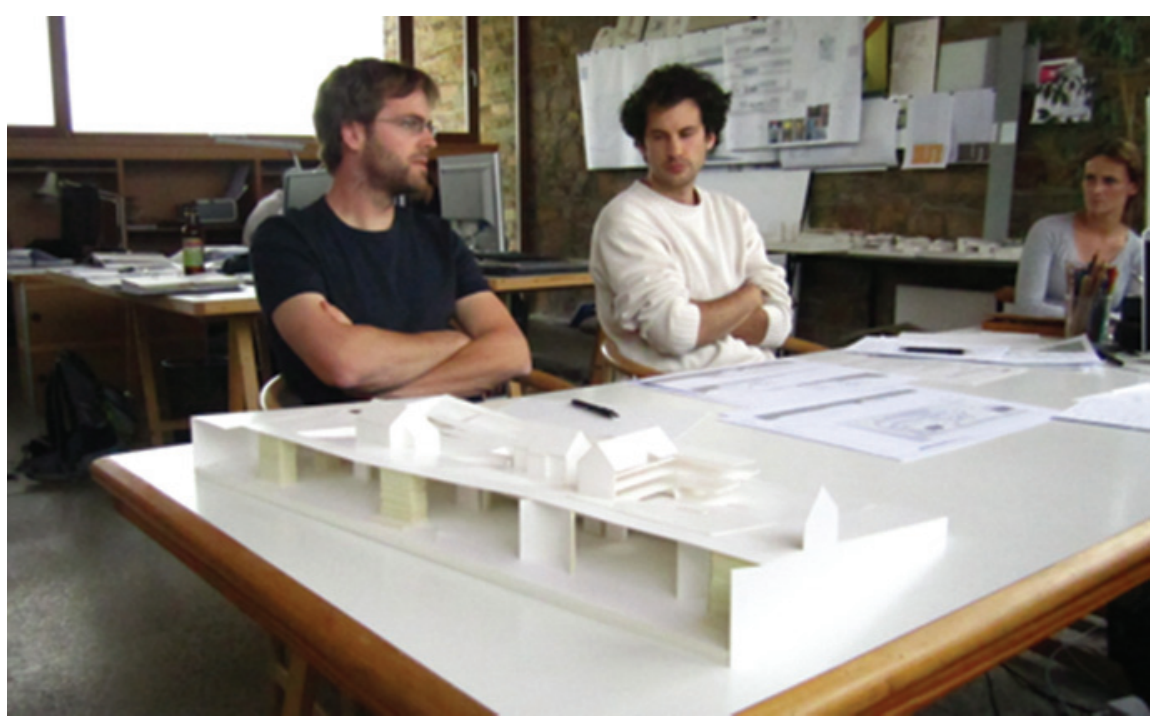

Figure 3 : Photo d'un moment de réflexion lors de la même réunion dans un atelier d'architecture en Belgique. 
La photographie peut également être définie comme une textualisation ponctuelle car elle segmente, extrait, ordonne et oriente l'action, se superpose à elle en sélectionnant certains instants. Mais, à différence des notes, la lecture d'une photo construit un parcours tabulaire car elle se fait par sauts analogiques entre les différents centres de l'attention et les différentes saillances perceptives.

3. La vidéo (Figure 4) peut apparaître comme la représentation la plus fidèle car elle suit le déploiement des actes, les accompagne et les restitue au travers du filtre d'un regard surplombant faisant émerger tous les gestes des acteurs en jeu.

Nous pourrions décrire la vidéo comme une textualisation durative et la définir comme un lieu de croisement de la lecture fondée sur le parcours linéaire et de la lecture fondée sur le parcours tabulaire. En effet, la vidéo associe la linéarité du mouvement de l'image dans le temps, qui trace un avant et un après, voire un développement, aux règles de la tabularité : la lecture linéaire croise la lecture de chaque photogramme, qui procède par sauts perceptifs. La lecture de la vidéo permet un croisement entre la lecture des formes qui se transforment tout au long du parcours audiovisuel (linéarité et horizontalité) et les lignes de force qui émergent de la relation entre la topologie du photogramme et les formes que ce dernier accueille (tabularité).

Si les trois textualisations que nous venons d'examiner sont autographiques car elles sont des résultats de gestes uniques et non répétables du corps, ${ }^{10}$ la notation par contre fonctionne de manière différente (Figure 5).

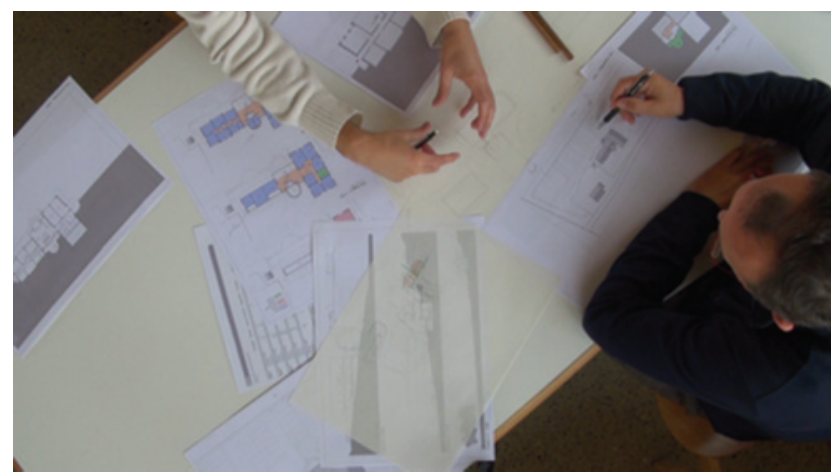

Figure 4 : Capture d'écran surplombant les opérations des architectes sur leur plan de travail.

10 Sur autographie et allographie, voir Goodman (2005 [1968]) et Dondero et Fontanille (2012). 


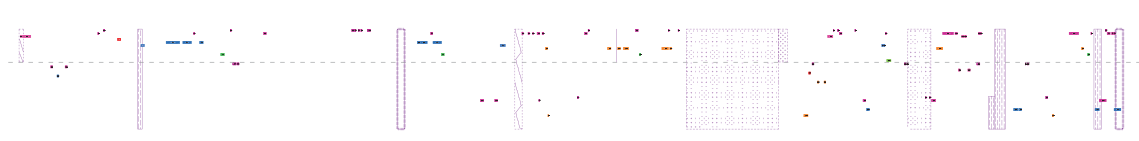

Figure 5 : Notation. Vision d'ensemble de la réunion à distance (55 min.). Encadrée en rouge une sélection de 5 min., agrandie dans l'image suivante (G. Joachim 2013).

Cette notation montre les différentes formes d'interaction graphique entre les architectes et la table digitale, leur répartition et leur distribution. Cette schématisation des activités graphico-gestuelles suit deux axes : l'axe vertical marque la position des interlocuteurs - la ligne en pointillés marque la séparation physique des deux équipes et l'axe horizontal marque un référent temporel. Les différentes interventions graphiques sont représentées par des segments dont la largeur indique la durée et dont la position correspond à un espace/temps précis de l'interaction (Figure 6).

Chaque segment est coloré selon la thématisation des actes graphicogestuels : le rouge correspond à des gestes de monstration. Le bleu correspond à la figuration d'un objet, l'orange à la figuration d'un mouvement et le vert à des interventions sur des rapports d'échelle, de proportion et de point de vue. Quant aux rectangles mauves, ils sont superposés sur le schéma pour signaler

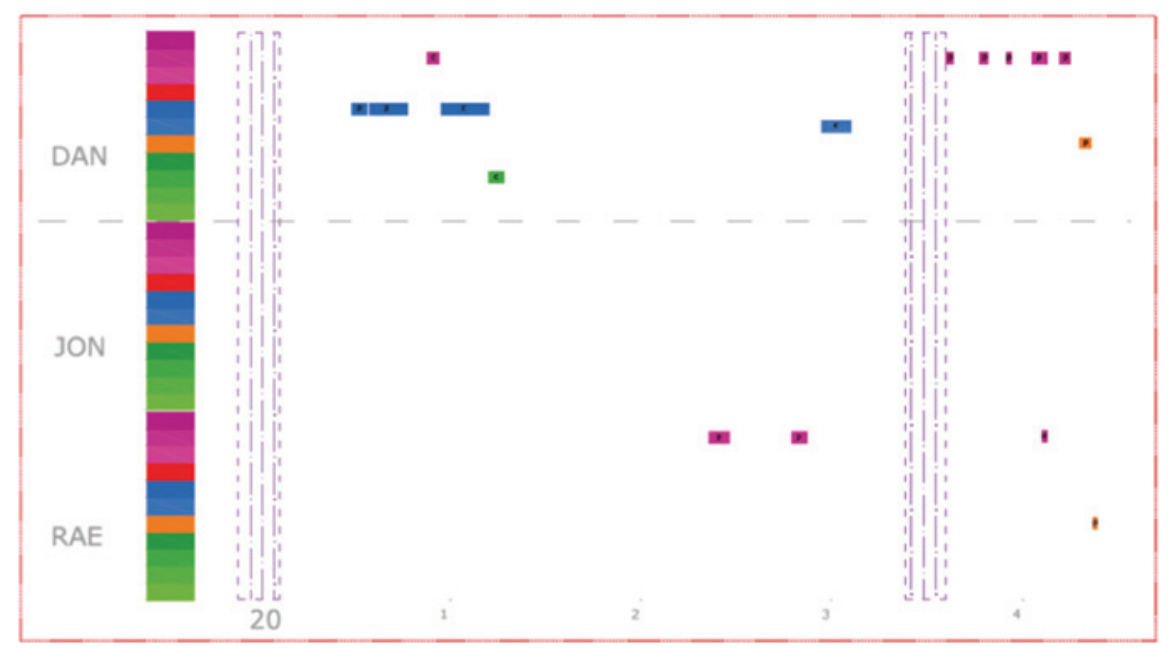

Figure 6 : Distribution des productions graphiques effectuées dans un espace de temps allant de la minute 20 à la minute 25 : dans ce temps, deux moments d'ajustement de l'affichage des documents sont verbalement explicités (en mauve dans la notation à la minute 20 et 23.30 ; Joachim 2013). 
les manipulations des documents graphiques et montrer les modifications de l'affichage des traits ainsi que de l'encadrement (agrandissement/réductions, etc.). ${ }^{11}$

Cette visualisation/formalisation des interventions graphiques sur la table graphique permet de faire émerger la répartition des actions des différents architectes, mais aussi de signaler si elles sont collectives ou individuelles.

La notation est la seule représentation de la pratique susceptible d'être définie comme allographique selon les termes de Goodman. Autrement dit, c'est une représentation qui, contrairement aux autres textualisations, ne mime ni suit le déploiement de l'action mais sélectionne ex-post les moments de pause, de coupure, de reprise mettant enfin en scène les modules qui reconstruisent la pratique à travers la constitution de modules d'action. La notation, non seulement visualise l'ensemble des gestes, mais met en scène ses rythmes et ses cadences grâce à la constitution d'homogénéités plus ou moins locales qui permettent la génération de modules, lesquels sont des unités d'action : la notation offre donc une visualisation de la grammaticalisation de la pratique.

\section{Conclusions}

Nous revenons ici sur la question que nous nous sommes posée au tout début : comment étudier la pratique sans en trahir la spécificité, sans la considérer comme un texte, et par conséquent en en valorisant l'organisation de sens ouvert ainsi que son caractère instable et éphémère ?

Résumons les étapes du parcours proposées tout au long de cette protoanalyse. La démultiplication et diversification des textualisations/visualisations permet à la pratique d'être apprivoisée dans ce qu'elle a d'éphémère (1) ; ensuite, elle permet d'être cartographiée par un croisement de points de vue et de spécificités médiumniques (2) ; pour finir, elle est re-dynamisée à travers la comparaison/traduction des différentes textualisations et la projection de l'une sur l'autre (3). L'action de projection d'une visualisation sur l'autre favorise la traduction intermédiatique à travers une analyse qui mime la pratique-objet d'analyse en reparcourant le croisement, non pas des actions, regards, échanges, mais de leur textualisations.

Une voie possible pour analyser la pratique à partir d'un point de vue impersonnel est par conséquent de construire une plateforme de traductions/

11 Pour une description plus détaillée, voir Joachim (2013). 
transpositions entre les différents types de textualisations (notes, vidéos, photos) et la notation. Les opérations de croisement et de traduction des textualisations $n+1$ permettent d'étudier la pratique comme quelque chose qui n'est pas clôturé avant le processus d'analyse. L'analyse, en effet, croise les textualisations, identifie les commensurabilités entre les unes et les autres, et met en scène les écarts entre les différentes formes de représentation/médiation. D'une certaine manière, l'analyse poursuit les textualisations en se dirigeant vers un processus de différentiation progressive des instances énonciatives.

\section{Références}

Angenot, Valérie, Maria Giulia Dondero, Guillaume Joachim \& Shima Shirkhodaei. 2013. Sémiotique de la communication en coprésence et à distance. Du textualisme à la sémiotique des pratiques. Numéro spécial, Interfaces numériques 2(3). 531-567.

Basso Fossali, P. (ed.). 2006. Testo, pratiche, immanenza. Numéro spécial, Semiotiche 5. Bordron, Jean-François. 2011. L'iconicité et ses images. Paris : PUF.

Bordron, Jean-François. 2014. Image et vérité. Essais sur les dimensions iconiques de la connaissance. Liège : PULg.

Dondero, Maria Giulia \& Jacques Fontanille. 2012. Des images à problèmes. Le sens du visuel à l'épreuve de l'image scientifique. Limoges : Pulim.

Eco, Umberto. 1975. Trattato di semiotica generale. Milan : Bompiani.

Eco, Umberto. 2013 [1984]. Sémiotique et philosophie du langage. Paris : PUF.

Elsen, Catherine \& Pierre Leclercq. 2008. SketSha ou le pouvoir de l'esquisse comme soutien de la conception collaborative. Lecture Notes in Computer Science 5220. 20-27.

Floch, Jean-Marie. 1990. Êtes-vous arpenteurs ou somnambules? L'élaboration d'une typologie comportementale des voyageurs du métro. In Sémiotique, marketing, et communication, 19-48. Paris : PUF.

Fontanille, Jacques. 2003. Sémiotique du discours. Limoges: Pulim.

Fontanille, Jacques. 2005. Du support matériel au support formel. In M. Arabyan \& I. KlockFontanille (eds.), L'ecriture entre support et surface, 183-200. Paris : L'Harmattan.

Fontanille, Jacques. 2008. Pratiques sémiotiques. Paris: PUF.

Fontanille, Jacques. 2014. L'énonciation pratique : Exploration, schématisation et transposition. In Communication multimodale et collaboration instrumentée : Regards croisés sur énonciations, représentations, modalités. http://www.lucid.ulg.ac.be/conferences/com mon14/downloads/Expose\%20Jacques\%20Fontanille.pdf (consulté le 20 mai 2017).

Fontanille, Jacques. 2015. Formes de vie. Liège : PULg.

Fontanille, Jacques \& Claude Zilberberg. 1998. Tension et signification. Liège: Mardaga.

Goodman, Nelson. 2005 [1968]. Langages de l'art: Une approche de la théorie des symboles, J. Morizot (trad.). Paris : Hachette.

Goodwin, Charles. 1994. Professional vision. American Anthropologist 96(3). 606-633.

Goodwin, Charles. 2000. Practices of seeing: Visual analysis. An ethnomethodological approach. In T. van Leeuwen \& C. Jewitt (eds.), Handbook of visual analysis, 157-182. London: Sage. 
Greimas, Algirdas Julien \& Joseph Courtés. 1979. Sémiotique. Dictionnaire raisonné de la théorie du langage. Paris : Hachette.

Hutchins, Edwin. 1995. Cognition in the wild. Cambridge: MIT Press.

Joachim, Guillaume. 2013. L'utilisation de représentations externes en conception architecturale collaborative médiée par ordinateur : le cas de la collaboration synchrone distante. Université de Liège : thèse de doctorat

Latour, Bruno. 2012. Enquête sur les modes d'existence. Paris : La Découverte.

Manetti, Giovanni. 1998. La teoria dell'enunciazione. Le origini del concetto e alcuni più recenti sviluppi. Sienne : Protagon.

Marrone, Gianfranco. 2010. L'invenzione del testo : Una nuova critica della cultura. Roma \& Bari : Laterza.

Metz, Christian. 1991. L'énonciation impersonnelle ou le site du film. Paris : Klincksieck.

Mondada, Lorenza. 1995. Chercheurs en interaction : Comment émergent les savoirs. Lausanne : Presses Polytechniques et Universitaires Romandes.

Paolucci, Claudio. 2010a. Enunciazione ed effetti di soggettività. In Strutturalismo e interpretazione, 433-500. Milan, Bompiani.

Paolucci, Claudio. 2010b. Quelques réflexions sur les énoncés: Textes, pratiques et cultures. Actes sémiotiques 113. http://epublications.unilim.fr/revues/as/1811 (consulté le 26 avril 2015).

Rajeb, Samia Ben \& Pierre Leclercq. 2013. Collaborations distantes synchrones dans les pratiques de conception. In Khaldoun Zreik \& Claude Yacoub (eds.), Echelles, espaces, temps : Ouvrage collectif regroupant les contributions présentées au 8e colloque international 01Design, 29-43. Paris : Europia.

Violi, Patrizia. 2006. Il soggetto è negli avverbi. Lo spazio della soggettività nella teoria semiotica di Umberto Eco. E/C. 